\title{
Microbiome disruption and recovery in the fish Gambusia affinis following exposure to broad-spectrum antibiotic
}

This article was published in the following Dove Press journal:

Infection and Drug Resistance

9 May 2017

Number of times this article has been viewed

\author{
Jeanette M Carlson' \\ Annie B Leonard' \\ Embriette R Hyde ${ }^{2,3}$ \\ Joseph F Petrosino $0^{2,3}$ \\ Todd P Primm' \\ 'Department of Biological Sciences, \\ Sam Houston State University, \\ Huntsville, ${ }^{2}$ Alkek Center for \\ Metagenomics and Microbiome \\ Research, ${ }^{3}$ Integrative Molecular and \\ Biomedical Sciences Training Program, \\ Department of Molecular Virology \& \\ Microbiology, Baylor College of \\ Medicine, Houston, TX, USA
}

\begin{abstract}
Antibiotics are a relatively common disturbance to the normal microbiota of humans and agricultural animals, sometimes resulting in severe side effects such as antibiotic-associated enterocolitis. Gambusia affinis was used as a vertebrate model for effects of a broad-spectrum antibiotic, rifampicin, on the skin and gut mucosal microbiomes. The fish were exposed to the antibiotic in the water column for 1 week, and then monitored during recovery. As observed via culture, viable counts from the skin microbiome dropped strongly yet returned to pretreatment levels by 1.6 days and became $>70 \%$ resistant. The gut microbiome counts dropped and took longer to recover ( 2.6 days), and became $>90 \%$ drug resistant. The resistance persisted at $\sim 20 \%$ of skin counts in the absence of antibiotic selection for 2 weeks. A community biochemical analysis measuring the presence/absence of 31 activities observed a 39\% change in results after 3 days of antibiotic treatment. The antibiotic lowered the skin and gut microbiome community diversity and altered taxonomic composition, observed by $16 \mathrm{~S}$ rRNA profiling. A 1-week recovery period did not return diversity or composition to pretreatment levels. The genus Myroides dominated both the microbiomes during the treatment, but was not stable and declined in abundance over time during recovery. Rifampicin selected for members of the family Comamonadaceae in the skin but not the gut microbiome. Consistent with other studies, this tractable animal model shows lasting effects on mucosal microbiomes following antibiotic exposure, including persistence of drug-resistant organisms in the community.
\end{abstract}

Keywords: microbiome, antibiotic, antibiotic resistance, Gambusia affinis, community disruption

\section{Introduction}

Antibiotics are potent tools in modern medicine, but select for resistance in pathogens and thus can become ineffective. An extensive study has been carried out and continues on drug-resistant mechanisms in pathogens. One area of research currently under intense investigation is the effects of antibiotic therapies on the normal microbiota of patients. Although the effects vary based on the particular antibiotic, the route of administration, and the composition of the preexisting microbial community, some general effects on human microbiota include lowering community diversity, change in community composition, loss of some rare members, alteration of enzymatic functions, selection of resistant members, and, in some but not all cases, lowering of total numbers. ${ }^{1}$ An antibiotic treatment in humans can have unintended consequences, such as diarrhea, antibiotic-associated enterocolitis, and opportunistic infections, most notably yeast and Clostridium difficile. In a study of three humans treated with ciprofloxacin, ${ }^{2}$ the gut microbiota was strongly and rapidly disrupted upon onset of treatment, and
Correspondence: Todd P Primm Department of Biological Sciences, Sam Houston State University, I 900 Avenue I, Huntsville, TX 7734I, USA

Tel +I 9362942689

Email tprimm@shsu.edu 
began to shift back to the initial state 1 week after cessation of the antibiotic. However, the composition did not return completely, instead obtained an alternative stable state. The drug treatment changed the abundance of approximately one-third of the gut taxa, and decreased the community richness, diversity, and evenness. ${ }^{3}$ In nine newborns, parenteral treatment with a combination of ampicillin and gentamicin at $<2$ days of age resulted in a decrease of the genera Bifidobacterium and Lactobacillus, and an increase in the class Proteobacteria, which persisted for at least 1 month. ${ }^{4}$

Studies in laboratory model organisms can be far more controlled and comprehensive, and complement clinical studies. Mice given two antibiotics and one biocide (ampicillin, metronidazole, and bismuth) in feed for 10 days demonstrated a dramatic shift in bacterial community composition in the cecea. $^{5}$ The composition was stable in the absence of antibiotic pressure. Although the normal gut microbiota of mice was dominated by the phyla Firmicutes (74.4\%) and Bacteroidetes $(23.3 \%)$, Proteobacteria took over (initially $1.1 \%$, changing to $75.5 \%$ ) after multidrug treatment. After a 2 -week recovery period, the gut returned almost completely to the initial composition. The treatment of mice with cefoperazone in the water for 10 days resulted in a less dramatic but still evident shift (loss of Bacteroidetes, increase in Firmicutes, and small change in Proteobacteria). Again, recovery was incomplete. Thus, the exact disruption of microbiome community composition caused by the antibiotics appears to vary according to each particular antibiotic and across model organisms.

This study examined the effect of a high dose of broadspectrum antibiotic on community composition, biochemical activities, and drug resistance in the skin and gut microbiomes of a vertebrate model, Gambusia affinis (the Western mosquito fish). These small (sizes of $0.1-1.5 \mathrm{~g}$ ) fish are a good model for mucosal microbiomes, ${ }^{6}$ easy to manipulate, and have vertebrate acquired immunity. Mucosal microbiomes have the highest densities of microbes and the most intimate interactions with the host. Rifampicin was chosen as a prototypical broad-spectrum antibiotic because the dominant resistance mechanism, substitutions in the beta subunit of the RNA polymerase ( $r p o B$ gene), is unlikely to be horizontally transferred between bacteria. It is also distributed well and evenly in tissues. Rifampicin, which is a primarily bactericidal member of the ansamycin family, has been used in human and veterinary medicine for many years, commonly against meningitis and mycobacterial infections. Because of rapid antibiotic accessibility and ability to sample without lethality to the host, this study focused on the skin mucosal microbiome. However, given that many published studies have examined the gut, this was included as well for comparison.

\section{Materials and methods}

\section{Fish}

For genetic homogeneity, the fish were caught from one location, a pristine pond in Walker County, Texas. Precautions were taken to avoid physically touching the fish by using a dip net, to not disturb the skin microbiome. The fish were maintained in $75 \mathrm{~L}$ aquaria with a 12-h light/12-h dark cycle until use (minimum 5-day acclimation to the laboratory after being caught) at $23^{\circ} \mathrm{C}-25^{\circ} \mathrm{C}$. For experiments, groups of fish were added to a well-cleaned plastic bucket into $2 \mathrm{~L}$ of autoclaved artificial pond water (APW). APW is of $0.33 \mathrm{~g} / \mathrm{L}$ $\mathrm{CaCl}_{2}, 0.33 \mathrm{~g} / \mathrm{L} \mathrm{MgSO}_{4}$, and $0.12 \mathrm{~g} / \mathrm{L} \mathrm{NaHCO}_{3}$ in deionized water (Milli-Q system). During experiments, the fish were fed daily with $5 \mathrm{mg} /$ fish of flake food (TetraFin) in the bucket. All animal experiments were performed under the approval of Institutional Animal Care and Use Committee's protocols 13-04-29-1018-3-01 and 14-04-17-1018-3-01.

\section{Antibiotic}

Rifampicin stock solution ( $50 \mathrm{mg} / \mathrm{mL}$ in dimethyl sulfoxide) was stored at $-20^{\circ} \mathrm{C}$, and $500 \mu \mathrm{L}$ stock added per liter of APW $(25 \mu \mathrm{g} / \mathrm{mL}$ final concentration). Rifampicin activity was measured daily during 1 week in APW at $25^{\circ} \mathrm{C}$ and did not change, as measured by the size of a zone of inhibition (ZOI) on an overnight nutrient agar (NA) plate of Bacillus megaterium from a $50 \mu \mathrm{L}$ sample of APW. Assuming the steady-state internal concentration in fish is close to the water concentration of the $\operatorname{drug}(25 \mu \mathrm{g} / \mathrm{mL})$ and a mean fish size of $1 \mathrm{~g}$ (weights varied between 0.25 and $1.5 \mathrm{~g}$ ), this would be equivalent to an approximate $25 \mathrm{mg} / \mathrm{kg}$ dose. By comparison, recommended human dosages are $10-20 \mathrm{mg} / \mathrm{kg},{ }^{7}$ so this is a high-dose model. Directly following a 3-day or 1-week exposure period, the remaining fish were transferred via dip net into a new tub habitat with fresh $1 \mathrm{~L}$ APW without rifampicin for recovery.

\section{Sampling}

To extract the skin microbiome, the fish were vortexed in a sterile $15 \mathrm{~mL}$ conical tube in $2 \mathrm{~mL}$ of PBST $(137 \mathrm{mM}$ $\mathrm{NaCl}, 10 \mathrm{mM}$ phosphate, and $0.1 \%$ Tween $20, \mathrm{pH} 7.4$ ) for 1 minute, pausing shortly every 10 seconds. To extract the gut microbiome, a fish was euthanized by snipping the nerve cord just behind the head, and then the entire gut canal was extracted by cutting just behind the mouth and just before 
the anus. The extracted gut was placed onto a sterile petri dish, and cut into short 1-2 mm sections. Gut contents from all the combined sections were extracted into $2 \mathrm{~mL}$ PBST by vortexing for 1 minute. For colony counts, tenfold serial dilutions of skin or gut extract were made in PBST, and then 100 $\mu \mathrm{L}$ aliquots spread onto NA (7.5 g/L of Bacto agar, $2.5 \mathrm{~g} / \mathrm{L}$ of peptone, and $1.5 \mathrm{~g} / \mathrm{L}$ of beef extract) plates in duplicate. NA containing $25 \mu \mathrm{g} / \mathrm{mL}$ rifampicin was used to quantify resistant organisms. The plates were checked after $48 \mathrm{~h}$ of incubation at $25^{\circ} \mathrm{C}$.

All data except the community biochemical profiles and antibiograms came from one experiment. Fifty fish from the same aquarium were placed in $2 \mathrm{~L}$ of APW with $25 \mu \mathrm{g} / \mathrm{mL}$ of rifampicin for 1 week, and then moved to another bucket with $2 \mathrm{~L}$ of fresh APW for recovery. Two fish were sampled for metagenomics (one for skin and one for gut) on every day of the 7-day treatment, and 1,2, 4, 6, and 14 days (skin only) afterward. Eight fish were sampled before treatment (four for skin and four for gut), and three aquarium water samples were taken at the same time. For a culture analysis, two fish (one for gut and one for skin) were sampled pretreatment, two fish on each day of the 7-day treatment, and two fish 7 and 14 days after treatment.

\section{Metagenomics}

For 16S rRNA gene profile analysis, bacteria were pelleted from each fish by a 2-minute spin in a centrifuge at room temperature at top speed $(13,000 \mathrm{rpm})$. The bacterial pellet was frozen and stored at $-80^{\circ} \mathrm{C}$, and then shipped on dry ice to the Alkek Center for Metagenomics and Microbiome Research, Baylor College of Medicine. Genomic DNA was extracted using the PowerSoil DNA Isolation Kit (MoBio Laboratories, Carlsbad, CA, USA) following protocols benchmarked during the Human Microbiome Project and the Earth Microbiome Project. The V4 region of the 16S rRNA was amplified using the $515 \mathrm{f} / 806 \mathrm{r}$ (barcoded) primer pair. ${ }^{8}$ Amplicons were pooled and sequenced in a single Illumina HiSeq run at the Human Genome Sequencing Center at Baylor College of Medicine.

Illumina reads 1 and 2 were quality filtered to remove any ambiguous bases and joined using a custom perl script with a minimum overlap of 15 base pairs (bps). Sequences were then quality trimmed, demultiplexed, and analyzed using the QIIME pipeline, version 1.8.0. ${ }^{9}$ Quality trimming used the following requirements: no ambiguous bases, maximum of one barcode error allowed, and a minimum phred score of 20. Median sequence length after trimming was $253 \mathrm{bp}$, and mean reads per sample were 204,325 . The sequences were then binned into operational taxonomic units (OTUs) using the closed-reference OTU picking workflow, with a sequence identity of $97 \%$ against the Greengenes (August 2012 release) as the reference database and UCLUST ${ }^{10}$ as the underlying clustering algorithm. Singleton reads were then removed from the OTU table, which was rarefied to 31,856 sequences per sample before performing alpha- and beta-diversity analyses.

\section{Community biochemical and resistance profiles}

The same method of extracting the skin microbiome from the fish done in the previous experiments was used, but with $5 \mathrm{~mL}$ of $0.85 \%$ saline solution instead of PBST. For the control, three untreated fish from the aquarium were separately extracted and analyzed using API-20E and API-20NE strips (bioMerieux). The strips were placed into an incubator at $25^{\circ} \mathrm{C}$, and read after $24 \mathrm{~h}$. For the antibiotic exposure analysis, three fish from the same aquarium were exposed to the antibiotic rifampicin at $25 \mu \mathrm{g} / \mathrm{mL}$ in APW for 3 days, and then sampled.

The same protocol using six fish (three controls and three treated) with 3 days of rifampicin exposure was used for the community resistance profile. The antibiogram was determined using the disk diffusion method.

\section{Results \\ Effects on number of culturable sensitive and resistant bacteria}

As shown in Figure 1A, the proportion of the skin bacterial population that was naturally resistant to rifampicin was very low (was below the detection limit of $\sim 20$ colonyforming units $[\mathrm{CFUs}] /$ fish), and this was consistent across multiple experiments (data not shown). Initiation of antibiotic exposure resulted in a rapid loss of viability for the skin microbiome (73-fold drop in the CFU numbers at $17.5 \mathrm{~h}$ ). However, culturable numbers were fully recovered and maintained after $38.5 \mathrm{~h}$. During the 1-week exposure to antibiotic, the CFU numbers on rifampicin-containing plates were $71 \%$ (1.6 days), $73 \%$ ( 2.7 days), and $88 \%$ (3.9 days) those of nutrient agar. Most of the culturable bacteria were drug resistant during the exposure, with the proportion being relatively stable. Following the release of antibiotic selection pressure, the culturable resistance rate dropped to $18 \%$, and was stable at that rate for at least 2 weeks (16.7 days was the last timepoint) in the absence of selection pressure.

Similarly, in the gut (Figure 1B), the resistance rate before exposure was very low $(0.02 \%)$, and the exposure resulted in a dramatic 13,036-fold drop in the CFU numbers at $17.5 \mathrm{~h}$. 
A

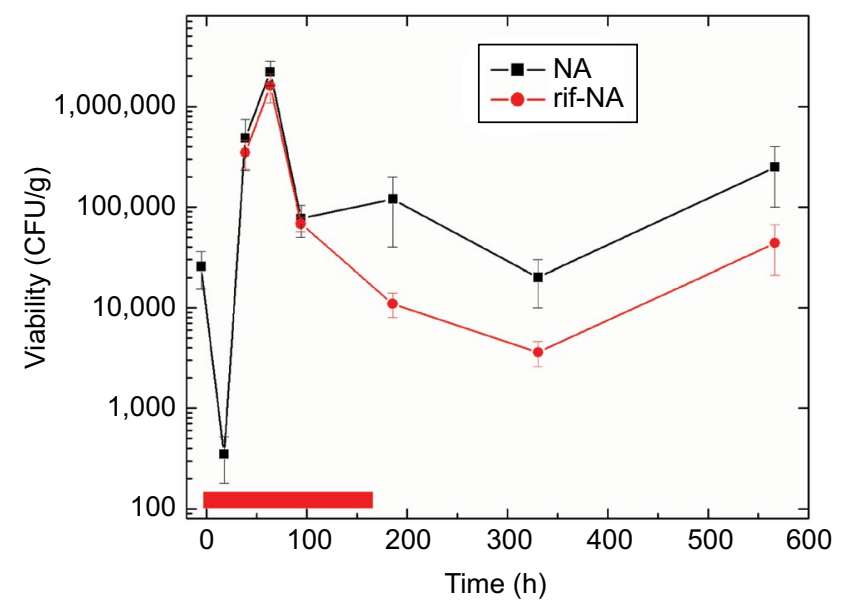

B

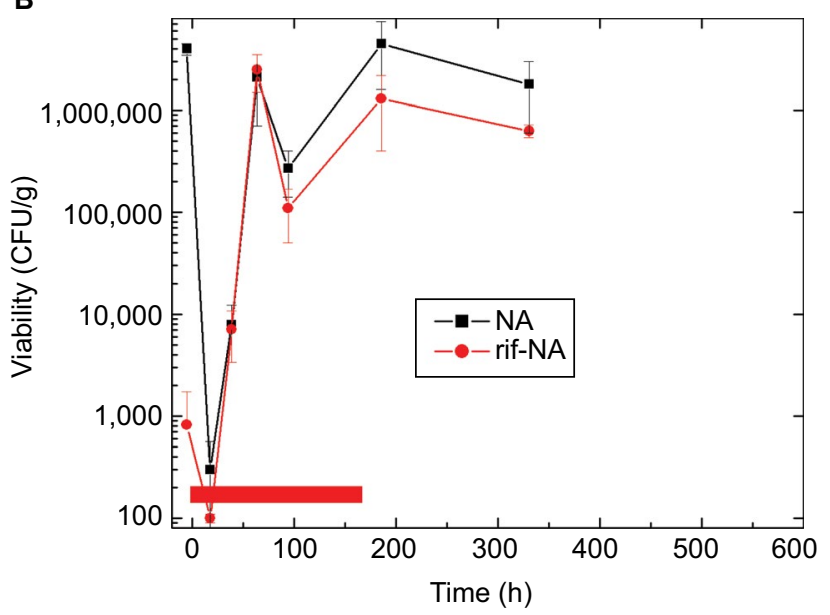

Figure I Culturable bacteria during antibiotic exposure. (A) Skin and (B) gut.

Notes: First samples are $5.5 \mathrm{~h}$ before rifampicin exposure began (control). Exposure started at time zero, and lasted $165 \mathrm{~h}$ (indicated by red bar), so the last three timepoints are $20.5,165.5$, and $400.5 \mathrm{~h}$ (skin only) after antibiotic removal by water change, respectively. No colonies were recovered on rifampicin-containing plates at the 5.5- and 17.5-h timepoints from skin. Scales of axes for panels (A) and (B) are identical, for ease of comparison. Viability is reported in CFUs/g of fish weight.

Abbreviations: CFU, colony-forming unit; NA, nutrient agar; rif-NA, rifampicin-containing NA.

Recovery was slower, with only $0.01 \%$ of the pretreatment colony numbers appearing at $38.5 \mathrm{~h}$, but numbers similar to pretreatment by $63.5 \mathrm{~h}$ and later. Assuming a slower exchange of bacteria between the fish gut and external environment than the fish skin and surrounding water, as well as a possible slower exchange of the antibiotic, this would be expected. Analogous with the skin, resistance rates were high during exposure ( $83 \%$ average of the three timepoints), and then dropped after the fish were moved to fresh drug-free water ( $29 \%$ at $185.5 \mathrm{~h}$ and $35 \%$ at $330.5 \mathrm{~h}$ ). Resistance rates also appeared to be stable, although they were only sampled out to 6.9 days posttreatment, with no gut timepoint taken at 16.7 days. Interestingly, the resistance rates of the gut posttreatment were almost double that of the skin, suggesting either a slower exchange with the environment or greater competitive fitness of the resistant species/strains. The preliminary bioassay data suggest that fish clear rifampicin from their body in $\sim 24 \mathrm{~h}$ (data not shown).

To confirm the resistance rates determined by comparison of CFU numbers on the NA plates to the NA plates containing rifampicin, one dozen colonies were selected at random from several NA plates from each timepoint. These colonies were then subcultured by streaking onto both NA and rifampicin-containing NA (rif-NA) plates, providing a separate determination of the proportion of resistant colonies that grew on the nonselective NA plates from the fish during exposure. The numbers determined were consistent. Although all 12 were resistant (regrew on NA and rif-NA plates) at both the skin and gut 38.5-h timepoints, only $40 \%$ of the gut and $17 \%$ of the skin colonies were resistant at the $330.5 \mathrm{~h}$ timepoint (6.9 days posttreatment).
One significant finding (in Figure 2) of the first major report $^{11}$ from the Human Microbiome Project was that the samples from the same body sites of different humans varied greatly with respect to phylogenetic diversity, yet were highly consistent with respect to presence of genes for certain metabolic pathways. This suggests that physiological functionality drives the microbiome community composition at each body site. Disruption of community biochemical activities may therefore better reflect microbiome dysfunction than taxonomic changes. ${ }^{12}$ Accordingly, biochemical tests were used to examine the pathways that were phenotypically active in the whole microbiome consortium before and after antibiotic exposure.

As expected for a complex community, the skin microbiome extracted before treatment was biochemically active, with 23 of 31 tests being positive (Table 1). Eight (35\%) of these enzyme activities were lost after 3 days of antibiotic exposure, whereas four new activities were gained. The 3-day timepoint was selected because it was the earliest timepoint at which the total number of culturable bacteria was very similar to pretreatment. When results from four of the tests that were duplicated between the API-20E and API-20NE systems conflicted, the positive result was assumed to be more reflective of the capabilities of the microbiome. Although host-derived enzymes in the skin mucus could potentially cause false positive results in these systems, it is unlikely since these enzymes would be at very low concentrations compared to the bacterial enzymes in this culture-based system.

To further examine the phenotypical properties of the skin microbiome consortium following antibiotic exposure, the susceptibility profile of the consortium was determined 


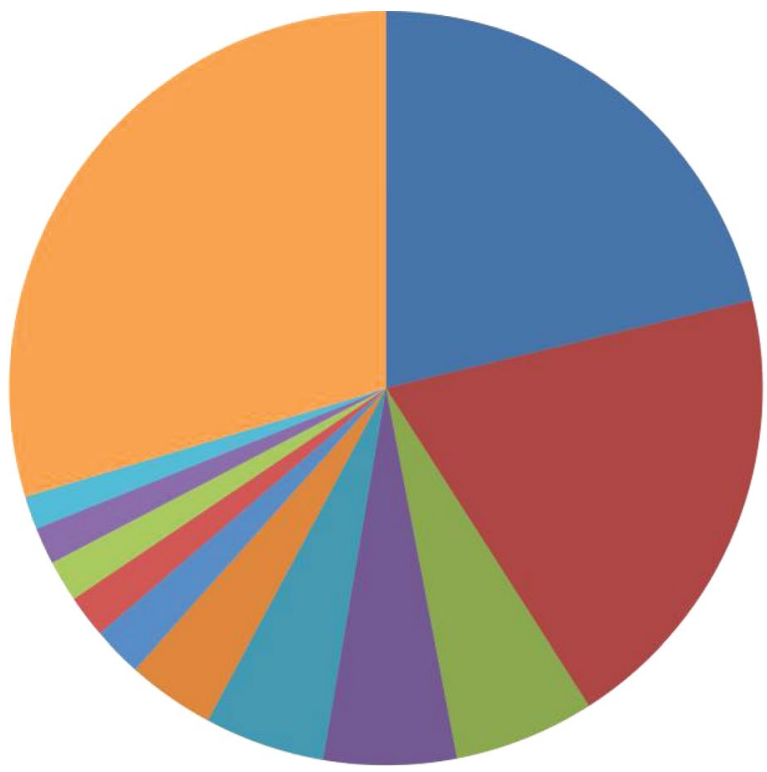

Massilia 21.2\%

Canditatus Captivus1 19.7\%

Rickettsia $6.0 \%$

Leptothrix 5.7\%

Ottowia $5.2 \%$

Candidatus Captivus2 3.8\%

Variovorax $2.1 \%$

FukuN18 1.8\%

Novosphingobium 1.8\%

B $1.6 \%$

LD28 1.4\%

Other $29.6 \%$

Figure 2 Wild-type skin microbiome composition.

Notes: Average percent normalized abundance of the top II taxa from skin of four control fish, shown at the genus level. "Candidatus" are candidate names not yet officially approved. Captivus is a proposed genus in the Holosporaceae family. Captivus I is identified as an "uncultured alpha proteobacterium," whereas the best match to Captivus2 sequences is identified as "uncultured bacterium." FukuN/8 is an uncultured bacterium within the class Spartobacteria, isolated from a lake in Germany. ${ }^{26}$ B is an unnamed genus within the family Comamonadaceae. LD28 is a genus within the family Methlophilaceae.

before and after 3 days of exposure (Table 2). Eighteen different antibiotics that target six different cellular pathways/ processes were used. As expected, the microbiota was sensitive to rifampicin before exposure but fully resistant afterward. No change was seen with five drugs (AMP, OXA, BAC, VAN, and G; antibiotic codes are given in Table 2), but the microbiome consortium was completely resistant to four of those both before and after rifampicin exposure. All of the four target cell wall synthesis, so it may be reflective of the almost exclusive Gram-negative composition of the skin community (Figures 2 and 3). Interestingly, the one other drug examined that targets cell wall synthesis was ceftriaxone, and RIF selection resulted in a switch from sensitive to fully resistant. Given the polymicrobial nature of the microbiome, the reason for this is unclear. For antibiotics (GEN, KAN, and PMB) in which the difference between the ZOI before and after exposure was less than the standard deviations of the two measurements, the results were not significant. Community susceptibility increased more than the measured ZOI standard deviations for two drugs, AMK and TET, which both target protein synthesis. The rifampicinselected microbiome community had a trend towards more sensitivity (larger ZOIs) to the aminoglycosides (AMK, GEN, KAN, and STR), which target the A-site on the 30 S subunit of the ribosome..$^{13,14}$ In contrast, erythromycin is a macrolide that targets the 50S subunit, which showed decreased susceptibility. Chloramphenicol, also targeting the 50S, showed decreased susceptibility. Although the microbiome was sensitive at the same level both before and after rifampicin selection against sulfisoxazole, at least some resistance to trimethoprim/sulfamethoxazole was selected.

The many susceptibility changes strongly suggest that a major change in the microbiome composition is caused by rifampicin selection. The DNA sequencing, via $16 \mathrm{~S}$ profiling, was used to confirm this. Given the inherent limitations of PCR amplification and next-generation sequencing with accurate identification and quantification of rare sequences, ${ }^{15}$ this analysis focused on the most abundant taxa $(\geq 1 \%$ of population).

The skin microbiota from untreated or fish exposed to rifampicin for 3 days was inoculated onto Mueller-Hinton plates with six antibiotic disks/plates and incubated at $25^{\circ} \mathrm{C}$ for $48 \mathrm{~h}$. The mean and standard deviation ZOI (in centimeters) for three fish from each condition are shown. The number in the code is the amount of antibiotic in the disk in micrograms, except for G-.25 (250 $\mu \mathrm{g})$ and SXT (1.25 $\mu \mathrm{g}$ of trimethoprim and $23.75 \mu \mathrm{g}$ of sulfamethoxazole).

Consistent with previous studies of animal microbiomes, the fish skin microbiome is diverse, but not equitable, being dominated by a few taxa. ${ }^{11,16,17}$ The dominant five taxa comprise $57.8 \%$ of all recovered sequences, and the top eleven are $70.4 \%$ of all sequences (Figure 2). In total, 648 Eubacterial taxa were recovered from the four control fish, with 419 of those taxa shared by at least two of the four fish. The consistency between fish was high, as only one taxa (18th-most abundant, Xanthomonas, lacking from one fish) within the 
Table I Biochemical culture results from skin microbiome samples

\begin{tabular}{|c|c|c|c|}
\hline Test & Activity & Control $^{\mathbf{a}}$ & Treat $^{\mathbf{b}}$ \\
\hline ONPG/PNPG & $\beta$-Galactosidase & + & + \\
\hline ADH/ADH & Arginine dihydrolase & + & $+1-$ \\
\hline LDC & Lysine decarboxylase & + & - \\
\hline ODC & Ornithine decarboxylase & - & - \\
\hline $\mathrm{CIT} / \mathrm{ClT}$ & Citrate use & - & $+1-$ \\
\hline $\mathbf{H} 2 \mathrm{H}$ & $\mathrm{H}_{2} \mathrm{~S}$ from thiosulfate & + & - \\
\hline URE/URE & Urease & - & - \\
\hline TDA & Tryptophane deaminase & + & + \\
\hline IND/TRPc & Indole production & + & - \\
\hline VP & Acetoin production & - & + \\
\hline GEL/GEL & Gelatinase & $-1+$ & - \\
\hline $\mathbf{G L U} / G L U^{d}$ & Glucose ox/ferm & + & + \\
\hline GLU & Glucose fermentation & + & - \\
\hline MAN/MAN & D-mannitol ox/ferm & + & + \\
\hline IND & Inositol ox/ferm & - & - \\
\hline SOR & D-sorbitol ox/ferm & - & + \\
\hline RHA & L-rhamnose ox/ferm & + & + \\
\hline SAC & D-saccharose ox/ferm & + & + \\
\hline MEL & D-melibiose ox/ferm & - & + \\
\hline AMY & Amygdaline ox/ferm & + & + \\
\hline ARA/ARA & L-arabinose ox/ferm & + & $+1-$ \\
\hline NO3 & Nitrate reduction & + & + \\
\hline ESC & Esculin $\beta$-glucosidase & + & + \\
\hline MNE & D-mannose ox/ferm & + & - \\
\hline NAG & $\mathrm{N}$-acetyl-glucosamine ox/ferm & + & + \\
\hline MAL & D-maltose ox/ferm & + & + \\
\hline GNT & Gluconate ox/ferm & + & + \\
\hline CAP & Capric acid ox/ferm & + & - \\
\hline$A D I$ & Adipic acid ox/ferm & + & - \\
\hline$M L T$ & Malic acid ox/ferm & + & + \\
\hline PAC & Phenylacetic acid ox/ferm & - & - \\
\hline
\end{tabular}

Notes: Results between all three fish in each group matched $100 \%$ of the time. Bold indicates the test result from the API-20E strip, and italics indicates the test result from the API-20NE strip. ${ }^{a}$ Control is three untreated fish. ${ }^{\text {b}}$ Treat is three fish after 3 days of rifampicin exposure. IND and TRP are two names for the same test. ${ }^{d}$ Glucose ox/ferm was positive in both API-20E and API 20NE before and after, but glucose fermentation was lost after exposure to air. Four tests that were shared between the two strips had conflicting results: ADH, CIT, GEL, and ARA.

Abbreviation: Ox/ferm, oxidation or fermentation of the respective carbon source.

58 most abundant (representing $91.5 \%$ of all sequences) not being shared among all four fish, whereas all of the 229 taxa identified from just one out of the four fish were rare (below the abundance level of $0.04 \%$ ). Fish from the same aquarium have very similar skin microbiome compositions.

Fish skin, fish gut, and lake water microbiome communities contain mostly the same families, but at strongly different abundances (Figure 3). Of the 308 total bacterial families recovered on the skin, 237 are shared between skin and water, representing $99.8 \%$ of the sequence abundance on skin. Of the 398 families in the gut, 242 are shared between the gut and skin, which is $98.5 \%$ of the gut sequence abundance. In contrast, only one family in the top eight most abundant is present in both the gut $(9.2 \%)$ and skin (1.8\%) microbiomes, which is FukuN18. These two microbiomes also only share one abundant order, the one containing FukuN18, which is Chthoniobacterales. For both skin and gut, all of the eight families are within the Gram-negative lineage. Two of the top eight skin-derived families are shared with the surrounding water, Comamonadaceae ( $6.0 \%$ of water and $18.3 \%$ of skin) and Methylophilaceae (2.2\% of water and $2.8 \%$ of skin). Only one water family, Sporichthyaceae, is a Gram-positive clade.

Rifampicin exposure causes dramatic changes in the skin microbiome composition. The top five genera that dominate (57.8\%) on fish skin before rifampicin introduction at the six exposure timepoints only comprise $17.9 \%, 1.2 \%, 12.2 \%$, $8.9 \%, 5.1 \%$, and $5.1 \%$ of the skin composition (Figure 4 ). The genus Myroides (in the family Flavobacteriaceae), a consistently minor component $(0.007 \% \pm 0.001 \%)$ before exposure, is selected during the first three timepoints, comprising $91.1 \%, 34.8 \%$, and $15.4 \%$ of the population. Myroides dominates the microbiome during the first 3 days of antibiotic selection, but is not stable. The top five genera that dominate after 7 days of antibiotic exposure $(28.7 \%$ Variovorax, $21.6 \%$ Hydrogenophaga, 7.5\% Mitsuaria, 7.2\% Pelomonas, and $3.9 \%$ Ottowia), only total $0.038 \%$ of the pretreated microbiome. Interestingly, four of those five genera are in the family Comamonadaceae (Mitsuaria is uncertain at family level, but in same order of Burkholderiales). Seven taxa increase in abundance in the majority of the treatment timepoints, especially at the end. These genera (Variovorax, Vibrio, Pelomonas, Hydrogenophaga, Pseudomonas, Mitsuaria, and family Spirochaeta) are apparently selected by the antibiotic.

Rifampicin selection also alters the gut microbiome composition. Of the 16 dominant OTUs shown in Figure 5, the first eight have a declining pattern (highest abundance in the untreated gut, lower at the 17.5-h timepoint, and least at the ending 115.5-h timepoint). These eight are abundant in the untreated gut (together $46.6 \%$ of all sequences), dropping to $19.6 \%$ at $17.5 \mathrm{~h}$, and at $115.5 \mathrm{~h}$ only compose $0.76 \%$ of all sequences, an approximately 60 -fold final decline. Rifampicin exposure thus strongly selects against this originally dominating group. Only two of the six gut samples collected during antibiotic exposure had an acceptable sequence quality. As was observed on the skin, the genus Myroides comes to dominate $(0.0054 \%$ pretreatment, $63.3 \%$ at $115.5 \mathrm{~h})$, although this effect is significantly delayed. Five of the OTUs (family Cetobacterium along with genera Variovorax, Myroides, Achromobacter, and Vibrio) have an increasing pattern (lowest abundance in the pretreatment condition, more at $17.5 \mathrm{~h}$, and then maximum at $115.5 \mathrm{~h}$ ). These five account for 
Table 2 Antibiogram of skin microbiome

\begin{tabular}{lllll}
\hline Untreated fish & Treated fish & Code & Antibiotic & Antibiotic target \\
\hline $2.57 \pm 0.12$ & $3.07 \pm 0.2$ & AMK-30 & Amikacin & Protein synthesis \\
$2.7 \pm 0.35$ & $3.17 \pm 0.15$ & GEN-10 & Gentamicin & \\
$2.6 \pm 0.17$ & $3.3 \pm 0.54$ & KAN-30 & Kanamycin & \\
$2.0 \pm 0.56$ & $2.37 \pm 0.25$ & STR-10 & Streptomycin & Chloramphenicol \\
$4.07 \pm 0.12$ & $2.33 \pm 0.42$ & CHL-30 & Tetracycline & Cell wall synthesis \\
$2.23 \pm 0.46$ & $3.33 \pm 0.06$ & TET-30 & Erythromycin & \\
$1.8 \pm 0.1$ & $1.27 \pm 0.32$ & ERY-15 & Ampicillin & \\
0 & 0 & AMP-10 & Ceftriaxone & \\
$4.07 \pm 0.38$ & 0 & CRO-30 & Oxacillin & Cell membrane \\
0 & 0 & OXA-I & Bacitracin & DNA gyrase \\
0 & 0 & BAC-10 & Vancomycin & \\
0 & 0 & VAN-30 & Polymyxin B & RNA polymerase \\
$1.97 \pm 0.15$ & $2.33 \pm 0.58$ & PMB-300 & Ciprofloxacin & Folic acid synthesis \\
$4.23 \pm 0.21$ & $2.9 \pm 0.36$ & CIP-5 & Nalidixic acid & \\
$4.4 \pm 0.17$ & $3.77 \pm 0.06$ & RIF-5 -30 & Rifampicin & \\
$1.9 \pm 0.1$ & 0 & G-.25 & Sulfisoxazole & Trimethoprim/sulfamethoxazole \\
$2.97 \pm 0.25$ & $3 \pm 0.01$ & SXT & & \\
$3.5 \pm 0.3$ & 0 & &
\end{tabular}

a total of $4.0 \%$ (with Cetobacterium being $3.4 \%$ by itself) of the control gut composition, yet together compose almost all (94.2\%) of the gut community at $115.5 \mathrm{~h}$. Overall, following the rifampicin treatment, the gut exhibits a clear selection away from the initial abundant community members to an almost completely different composition. Although the skin is dominated by the family Comamonadaceae (top five genera totaling $68.9 \%$ ) at the last 161.5-h timepoint (and already having $32.4 \%$ at $115.5 \mathrm{~h}$ ), the gut is not at $115.5 \mathrm{~h}$ (contains only the abundant genus Variovorax at $15.5 \%$ ).

Selection by rifampicin has a similar effect on the gut and skin microbiomes. Comparing the two 115.5-h timepoints (Tables 3 and 4), the most abundant genus is the same, Myroides (63.3\% of gut and $15.4 \%$ of skin). Six of the eight most abundant genera in the gut are also present within the 30 most abundant genera of the skin. Although the skin only has 87 genera present, 68 of those are shared with the gut (contains 275 genera) at the same timepoint.

Rifampicin exposure causes a loss of over half of the OTUs from the skin after $50.5 \mathrm{~h}$ and thus a major decrease in diversity (Table 3 ). There is only a small return of diversity during the week of recovery. The proportions of the most abundant organisms likewise change during exposure with little return to the pretreatment state during recovery. The effects are also clear at the level of order (two taxonomic levels above genus). Before exposure, the four fish contain 172 different orders among them (gamma diversity), with 63 of those (37\%) being present on the skin of all four fish. Forty-nine orders were recovered from one of the fish only, yet they are all rare (highest abundance of $0.05 \%$ ). In contrast, from the six treated fish, only 116 orders were recovered, with only 30 present $(26 \%)$ in all six fish, and 37 found on one fish only (top abundance of $0.1 \%$ ). During the 1-week recovery after the antibiotic, the diversity loss continued, with only 85 orders being recovered from the five sampled fish. Thirty-one of those orders were present (36\%) on all five fish, and 22 on one fish only (highest abundance of $0.01 \%$ ).

The microbiome of the gut is affected more severely than that of the skin (Table 4). Before exposure, the gut microbiome of individual fish is more diverse (higher number of OTUs) and is more even (more OTUs with $\geq 0.1 \%$ abundance and the 20 most abundant OTUs making a smaller proportion of the total). The gut microbiome loses even more diversity (48\% of OTUs lost) during rifampicin exposure, and this does not return but continues to decline during the 1-week recovery period. The composition of abundant microbes also changes even more than the skin. Neither the skin microbiomes nor the gut microbiomes return to the pretreatment composition during the recovery period, and recovery communities are more similar to the treated than the untreated (Figure 6).

\section{Discussion}

To date, this is the most extensive study of antibiotic effects on the microbiome using a fish model. This is also the first study specifically of rifampicin. The gut microbiome of captive and wild-caught zebra fish (Danio rerio) contains similar genera ${ }^{16}$ as the mosquito fish gut, but at different abundances. The dominant genera from three zebra fish were Aeromonas (average abundance of 29.2\%), Streptophyta (13.5\%), Cetobacterium (13.3\%), Pseudomonas (10.4\%), 


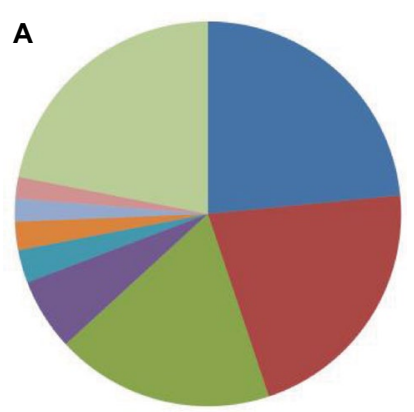

- Canditatus Captivus 23.5\%

- Oxalobacteraceae $21.4 \%$

= Comamonadaceae 18.3\%

— Rickettsiaceae $6.0 \%$

- Methylophilaceae 2.8\%

= Sphingomonadaceae 2.4\%

- Flavobacteriaceae $1.9 \%$

- FukuN18 1.8\%

- Other $21.9 \%$

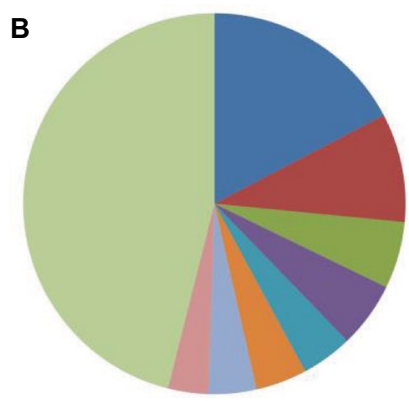

- Aeromonadaceae 17.3\%

- FukuN18 9.2\%

॥Planctomycetaceae $5.7 \%$

. Chitinophagaceae 5.6\%

- Rhodobacteraceae $4.3 \%$

= Rhodocyclaceae $4.3 \%$

- Neisseriaceae $4.0 \%$

I Fusobacteriaceae 3.4\%

॥ Other $46.1 \%$

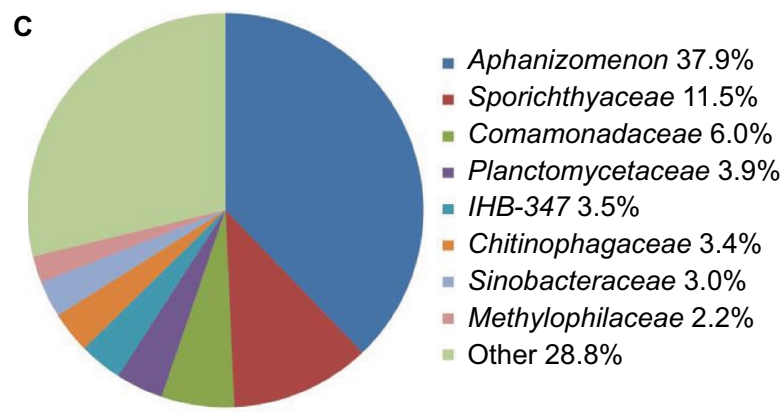

Figure 3 Comparison of fish skin, fish gut, and water microbiomes. (A) Eight most abundant families from fish skin (average normalized abundance from four fish). (B) Eight most abundant families from fish gut (average normalized abundance from four fish). (C) Eight most abundant families from water (average normalized abundance from two separate water samples).

Notes: Average percent abundances shown next to family name. IHB-347 is within phylum Cyanobacteria.

and Vibrio (4.7\%), with the four control fish exhibiting $17.3 \%, 0 \%, 3.4 \%, 0.6 \%$, and $0.08 \%$ of those genera, respectively. It should be noted that Streptophyta is in the class Cyanobacteria, and Gambusia fish gut contained 3.3\% of an uncultured Cyanobacteria, unclassified at the genus and family level. Zebra fish (family Cyprinidae) and mosquito fish (family Poeciliidae) do not join taxonomically until the level of order, Cyprinodontiformes, and thus are somewhat genetically distant, with host factors potentially selecting for different gut microbiota compositions. A significant physiological and anatomical difference also exists; zebrafish lay eggs, whereas mosquitofish have internal fertilization and give live birth.

Although most of the same genera are shared between the Gambusia skin and the gut, the dominant organisms in high abundance are very different. And although in fish both environments are mucosal surfaces, one likely major difference is oxygen level, with the skin being more aerobic and the gut more anaerobic. Of the abundant families in the gut, Aeromonadaceae and Rhodobacteraceae are facultative anaerobes, Rhodocyclaceae contains both facultative and obligate anaerobes, Planctomycetaceae, Chitinophagaceae, and Neisseriaceae are obligate aerobes, and Fusobacteriaceae contains obligate anaerobes. FukuN18 is uncultured and the metabolic classification is unknown. Of the abundant skin families, Oxalobacteraceae includes both obligate aerobes and anaerobes, Comamonadaceae, Sphingomonadaceae, and Methylophilaceae are obligate aerobes, and Flavobacteriaceae includes obligate aerobes and microaerobic species. Rickettiaceae are obligate intracellular organisms. Captivus is uncultured and has unknown oxygen requirements.

A previous study on the Gambusia skin microbiome suggested that this mucosal surface may be selective, not simply a passive recipient of bacteria from the water column. ${ }^{6}$ The differences in community composition between the skin and water observed in this study (Figure 3) support this conclusion.

Disruption of the fish skin microbiome by rifampicin results in only a transient $(<38.5 \mathrm{~h})$ loss of culturable bacteria; however, drug-resistant bacteria are rapidly selected for and persist for at least 2 weeks following the removal of antibiotic. Not only is the taxonomic distribution of the skin bacterial community strongly altered, the biochemical functions (of the culturable fraction) are also altered ( $43 \%$ change in results, 12 of 28 tests) during exposure. The biochemical function is not measured in most studies of antibiotic effects on microbiomes, so this interesting potential functional difference needs further investigation. The extent of gut microbiome loss of culturability is greater than the skin, and takes longer to recover (63.5 h). Antibiotic exposure not only changes the skin and gut community composition, but also reduces the overall diversity. Neither the microbiome (gut or skin) diversity nor the composition returns to pretreatment values during the 1-week recovery period without antibiotic. Grouping of communities on principal components analysis (Figure 6) suggests the communities stabilize to a different composition during recovery. It remains to be seen if, given more time, the communities would return to the pretreatment composition. In addition to the loss of diversity, the evenness is also reduced during antibiotic treatment (Table 3; rise in percent abundance of the Top 20), and drop in both $1 \%$ abundance ( $\mathrm{Ab}$ ) and $0.1 \%$ $\mathrm{Ab})$. Rifampicin also causes a major loss of diversity and evenness in the gut microbiome (Table 4). Treatment of salmon with oxytetracycline in food for up to 25 days did 


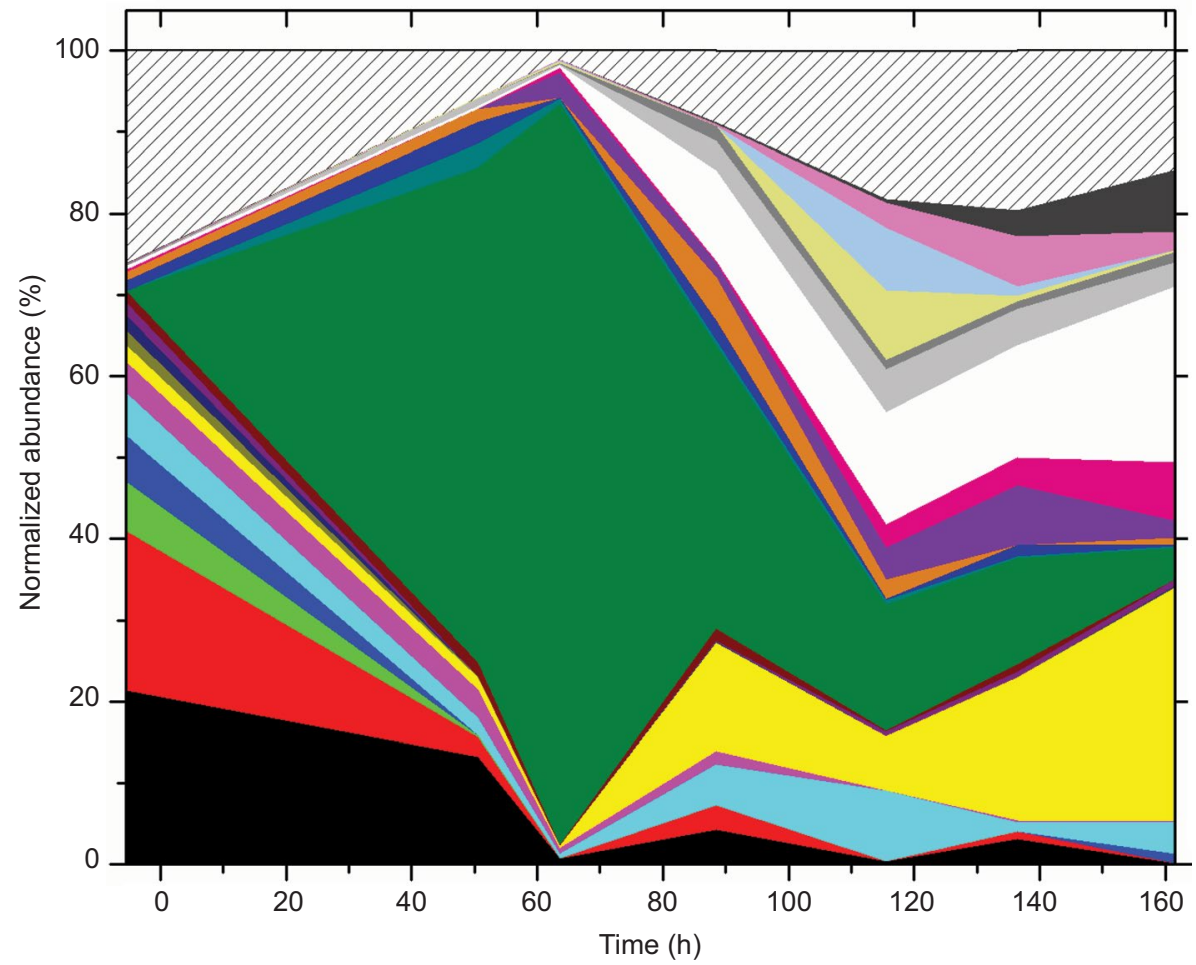

\begin{tabular}{|l|}
\hline Massilia \\
Captivus1 \\
Rickettsia \\
Leptothrix \\
Ottowia \\
Captivus2 \\
Variovorax \\
A \\
Novosphingobium \\
B \\
LD28 \\
Myroides \\
Cetobacterium \\
Polynucleobacter \\
Xanthomonas \\
Vibrio \\
Pelomonas \\
Hydrogenophaga \\
Spirochaeta \\
Tepidicella \\
Enterobacte \\
Shewanella \\
Pseudomonas \\
Mitsuaria \\
Other \\
\hline
\end{tabular}

Figure 4 Antibiotic effect on skin microbiome.

Notes: Abundance of dominant genera across time during the week of antibiotic exposure. At $5.5 \mathrm{~h}$ is represented the average of four fish pre-exposure. Exposure timepoints are at $50.5,63.5,88.5,115.5,136.6$, and $161.5 \mathrm{~h}$.

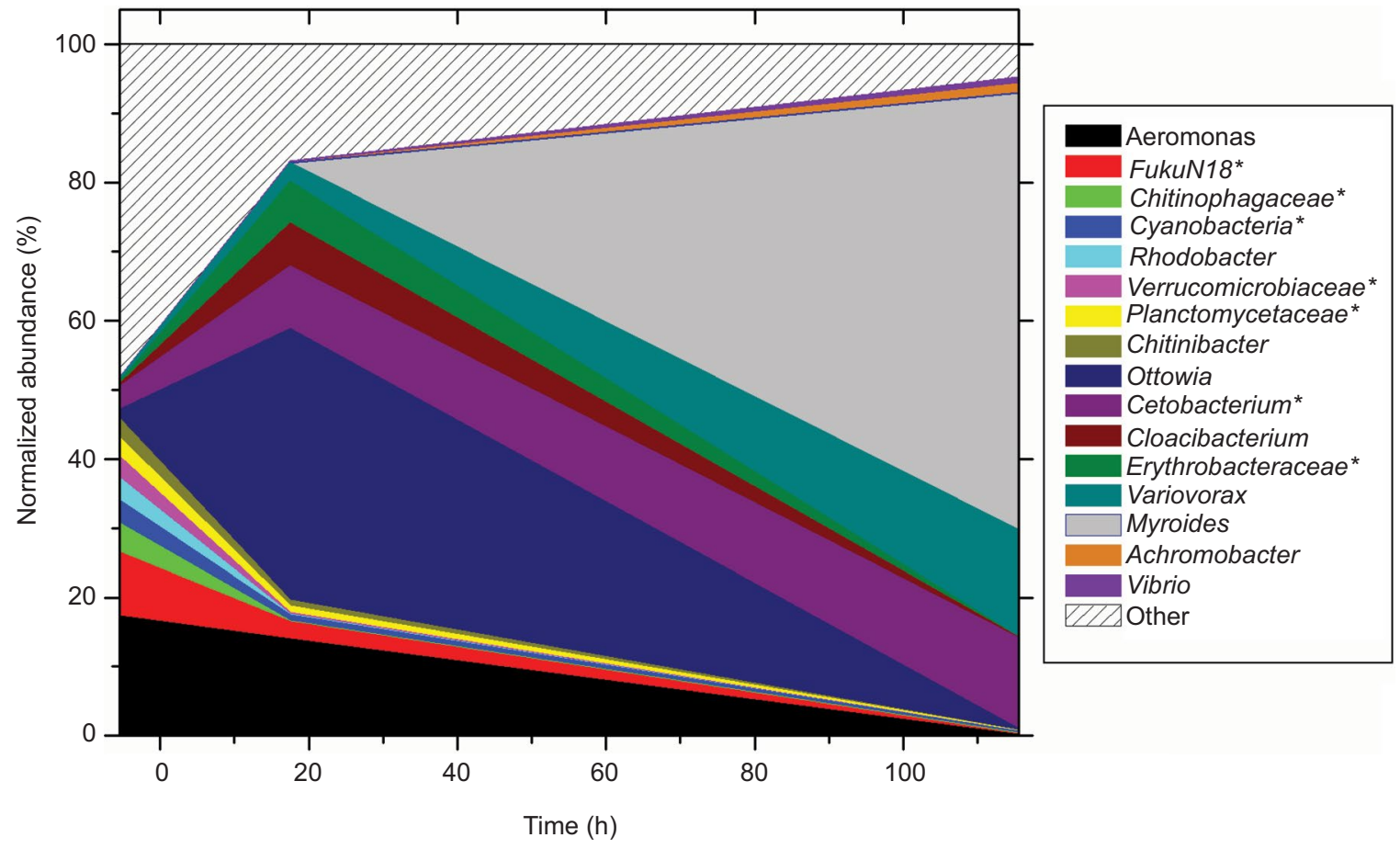

Figure 5 Antibiotic effect on gut microbiome.

Notes: Abundance of dominant genera across time during the week of antibiotic exposure. At $5.5 \mathrm{~h}$ is represented the average of four fish pre-exposure. Exposure timepoints are at 17.5 and 115.5 h. *For operational taxonomic units, where the genus is uncertain, the family name is given, except for Cyanobacteria, which is a phylum.

not alter overall plate counts from intestine, but did select for culturable resistant organisms and reduced community diversity as measured by RFLP. ${ }^{18}$
At $115.5 \mathrm{~h}$ of treatment, the genera present on the gut and skin overlap more than the pretreatment state. This may be due to an initial rapid depletion of skin organisms, and 
Table 3 Skin microbiome diversity before, during, and after treatment

\begin{tabular}{|c|c|c|c|c|c|c|c|}
\hline Timepoint & $\boldsymbol{\alpha}^{\mathbf{a}}$ & Top 20 & $\mathbf{I} \% \mathbf{A b}$ & $0.1 \% \mathbf{A b}$ & Dominate & $\beta$ & Relate \\
\hline $\mathrm{Cl}$ & 294 & $90.6 \%$ & 12 & 46 & 32.3\% Captivus I, 24.7\% Massilia, 7.0\% Captivus2 & - & - \\
\hline $\mathrm{C} 2$ & 345 & $80.9 \%$ & 18 & 79 & 23.6\% Massilia, I2.1\% Ottowia, 9.0\% Captivus I & - & - \\
\hline $\mathrm{C} 3$ & 414 & $78.7 \%$ & 19 & 82 & 19.0\% Massilia, 17.0\% Captivus I, 6.8\% Ottowia & - & - \\
\hline $\mathrm{C4}$ & 471 & $80.5 \%$ & 18 & 71 & 20.5\% Captivus I, 17.7\% Massilia, 7.2\% Rickettsia & - & - \\
\hline AvgC & $38 I \pm 78$ & $80.1 \%$ & $17 \pm 3$ & $70 \pm 16$ & 21.2\% Massilia, 19.7\% Captivus I, 6.0\% Rickettsia & 0 & $80.1 \%$ \\
\hline T50.5 & 155 & $97.0 \%$ & 11 & 22 & 60.8\% Myroides, 13.1\% Massilia, 3.5\% Captivus2 & 9 & $30.0 \%$ \\
\hline T63.5 & 64 & $99.4 \%$ & 2 & 16 & 91.1\% Myroides, 3.1\% Vibrio, 0.7\% Captivus2 & 12 & $2.5 \%$ \\
\hline T88.5 & 75 & $94.9 \%$ & 14 & 33 & 34.8\% Myroides, 13.4\% Variovorax, II.2\% Hydrogenophaga & 10 & $39.0 \%$ \\
\hline TII5.5 & 87 & $93.1 \%$ & 15 & 44 & I5.4\% Myroides, 13.8\% Hydrogenophaga, 8.7\% Ottowia & 14 & $21.7 \%$ \\
\hline TI36.5 & 136 & $91.0 \%$ & 17 & 39 & 17.5\% Variovorax, 13.8\% Hydrogenophaga, 13.0\% Myroides & 13 & $26.6 \%$ \\
\hline TI6I.5 & 128 & $91.0 \%$ & 15 & 43 & 28.7\% Variovorax, 21.6\% Hydrogenohaga, 7.5\% Mitsuaria & 14 & $37.0 \%$ \\
\hline AvgT & $108 \pm 37$ & $90.6 \%$ & $12 \pm 5$ & $33 \pm 12$ & $36.5 \%$ Myroides, II.3\% Variovorax, 10.2\% Hydrogenophaga & $12 \pm 2$ & $26.1 \%$ \\
\hline $\mathrm{R} 20.5$ & 166 & $98.1 \%$ & 12 & 23 & 46.7\% Comamonas, $22.1 \%$ Vibrio, $8.5 \%$ Pseudomonas & 12 & $7.7 \%$ \\
\hline R44.5 & 180 & $97.1 \%$ & 13 & 26 & $37.5 \%$ Variovorax, $13.9 \%$ Pseudomonas, $12.6 \%$ Comamonas & 14 & $54.6 \%$ \\
\hline R68.5 & 184 & $98.2 \%$ & 13 & 24 & $34.9 \%$ Variovorax, $10.6 \%$ Flavobacterium, $9.7 \%$ Hydrogenophaga & 16 & $46.5 \%$ \\
\hline $\mathrm{R} / 20.5$ & 148 & $97.4 \%$ & 14 & 29 & 24.0\% Mitsuaria, I8.8\% Flavobacterium, I4.0\% Hydrogenophaga & 15 & $27.1 \%$ \\
\hline $\mathrm{RI} 65.5^{\mathrm{b}}$ & 153 & $94.7 \%$ & 13 & 33 & 38.0\% Flavobacterium, 9.9\% Pseudomonas, 9.7\% Mitsuaria & 17 & $44.2 \%$ \\
\hline AvgR & $166 \pm 16$ & $95.2 \%$ & $13 \pm 0.7$ & $27 \pm 4$ & 17.7\% Variovorax, 15.0\% Comamonas, I4.1\% Flavobacterium & $15 \pm 2$ & $33.5 \%$ \\
\hline
\end{tabular}

Notes: All numbers are based on genus-level identification of sequences. Top 20 is the cumulative percent abundance of the 20 most abundant taxa. I\% Ab is the number of taxa with an abundance of $\geq 1 \%$, whereas $0.1 \% A b$ is the number of taxa with abundance of $\geq 0.1 \%$. Dominate is the percent abundance and identity of the three most abundant genera. $\beta$ is the absence/presence difference in the top 20 taxa between the sample and the average of the control samples (AvgC). Relate is the cumulative percent abundance of the same 20 taxa that are most abundant in the average of the control samples, thus how related the dominant taxa are to the pretreatment samples.

${ }^{a} \alpha$ is richness, the total number of OTUs in the sample. ${ }^{\circ}$ For the RI65.5 timepoint, if the abundance of Flavobacterium is subtracted, the relate value drops to $6.2 \%$. C and a number, the four control fish before treatment; $R$ followed by a number, the hours of recovery after removal of rifampicin; $T$ and a number, the hours of rifampicin treatment. Abbreviations: Ab, abundance; AvgC, mean and standard deviation of the controls; AvgR, mean and standard deviation of the recovery samples; AvgT, mean and standard deviation of the six treatment samples; OTU, operational taxonomic unit.

Table 4 Gut microbiome diversity before, during, and after treatment

\begin{tabular}{|c|c|c|c|c|c|c|c|}
\hline Timepoint & $\boldsymbol{\alpha}^{\mathbf{a}}$ & Top 20 & $\mathbf{I} \% \mathbf{A b}$ & $0.1 \% \mathbf{A b}$ & Dominate & $\boldsymbol{\beta}$ & Relate \\
\hline$\overline{\mathrm{GI}}$ & 538 & $64.7 \%$ & 23 & 99 & 12.9\% Aeromonas, 7.4\% FukuN/8, 5.3\% Rhodobacter & - & - \\
\hline G2 & 508 & $75.4 \%$ & 14 & 80 & 26.3\% Aeromonas, $10.7 \%$ FukuN I8, $10.2 \%$ Cetobacterium & - & - \\
\hline G3 & 436 & $61.2 \%$ & 21 & 111 & $15.6 \%$ Aeromonas, $7.2 \% \times I^{\mathrm{b}}, 3.6 \%$ Rhodobacter & - & - \\
\hline G4 & 402 & $74.4 \%$ & 22 & 80 & $16.5 \%$ FukuN $18,14.5 \%$ Aeromonas, $5.1 \%$ IHB-347c & - & - \\
\hline AvgG & $47 I \pm 63$ & $65.5 \%$ & $20 \pm 4$ & $93 \pm 15$ & $17.3 \%$ Aeromonas, $9.2 \%$ FukuN $18,4.2 \% \times I^{b}$ & 0 & $65.5 \%$ \\
\hline gTI7.5 & 168 & $89.5 \%$ & 10 & 58 & 39.3\% Ottowia, $14.0 \%$ Aeromonas, $9.0 \%$ Cetobacterium & 8 & $68.1 \%$ \\
\hline gTII5.5 & 282 & $97.6 \%$ & 4 & 21 & 63.3\% Myroides, 15.5\% Variovorax, 13.2\% Cetobacterium & 7 & $14.2 \%$ \\
\hline AvggT & 225 & & 7 & 40 & 31.7\% Myroides, 19.7\% Ottowia, II.1\% Cetobacterium & 7 & $41.7 \%$ \\
\hline gR20.5 & 218 & $97.3 \%$ & 8 & 23 & 47.5\% Variovorax, II.3\% Hydrogenophaga, II.3\% Cetobacterium & 3 & $12.0 \%$ \\
\hline gR68. $5^{d}$ & 142 & $99.2 \%$ & 5 & 17 & 65.2\% Cetobacterium, 19.9\% Variovorax, 5.65\% Shewanella & 1 & $65.4 \%$ \\
\hline$g R \mid 20.5^{d}$ & 96 & $99.8 \%$ & 5 & 14 & 69.8\% Cetobacterium, 19.9\% Variovorax, 5.65\% Shewanella & 3 & $75.5 \%$ \\
\hline gRI65.5 & 234 & $92.4 \%$ & 17 & 42 & 34.7\% Oryza, 17.8\% Flavobacterium, 10.5\% Mitsuaria & 3 & $2.9 \%$ \\
\hline AvggR & $173 \pm 65$ & $96.1 \%$ & $9 \pm 6$ & $24 \pm 13$ & $36.7 \%$ Cetobacterium, $17.4 \%$ Variovorax, $5.7 \%$ Shewanella & 2 & $65.5 \%$ \\
\hline
\end{tabular}

Notes: All numbers are based on genus-level identification of sequences. Top 20 is the cumulative percent abundance of the 20 most abundant taxa. $1 \%$ Ab is the number of taxa with an abundance of $\geq 1 \%$, whereas $0.1 \% \mathrm{Ab}$ is the number of taxa with abundance of $\geq 0.1 \%$. Dominate is the percent abundance and identity of the three most abundant genera. $\beta$ is the absence/presence difference in the top 20 taxa between the sample and the average of the control samples (AvgC). Relate is the cumulative percent abundance of the same 20 taxa that are most abundant in the average of the control samples, thus how related the dominant taxa are to the pretreatment samples. ${ }^{a} \alpha$ is richness, the total number of OTUs in the sample. ${ }^{b} \mathrm{X} I$ is an uncultured genus from the family Chitinophagaceae. ${ }^{\mathrm{c}} I \mathrm{HB}-347$ is an organism of indeterminate placement in the phylum Cyanobacteria. If the one genus Cetobacterium is removed from the analysis, then the relate value for sample gR68.5 is $0.2 \%$ and for gR I 20.5 is $5.7 \%$. G and a number, the four control fish before treatment; $g R$ followed by a number, the hours of recovery after removal of rifampicin; $g T$ and a number, the hours of rifampicin treatment.

Abbreviations: Ab, abundance; AvgG, mean and standard deviation of the controls; AvgT, mean (standard deviation cannot be obtained from two numbers) of the two treatment samples; AvggT, mean of two treatment samples (SD cannot be determined from less than 3 samples); OTU, operational taxonomic unit.

then recolonization of the skin by organisms from the gut via feces through the water column. It should be noted that in a recent study on rainbow trout, about $50 \%$ of the microbiome community diversity was present within the skin epithelium, so this may be serving as a major reservoir to reseed the mucosa, and was not examined in this study. ${ }^{19}$

Rifampicin-resistant organisms are likely present at low levels in the Gambusia skin microbiome (the detection limit 


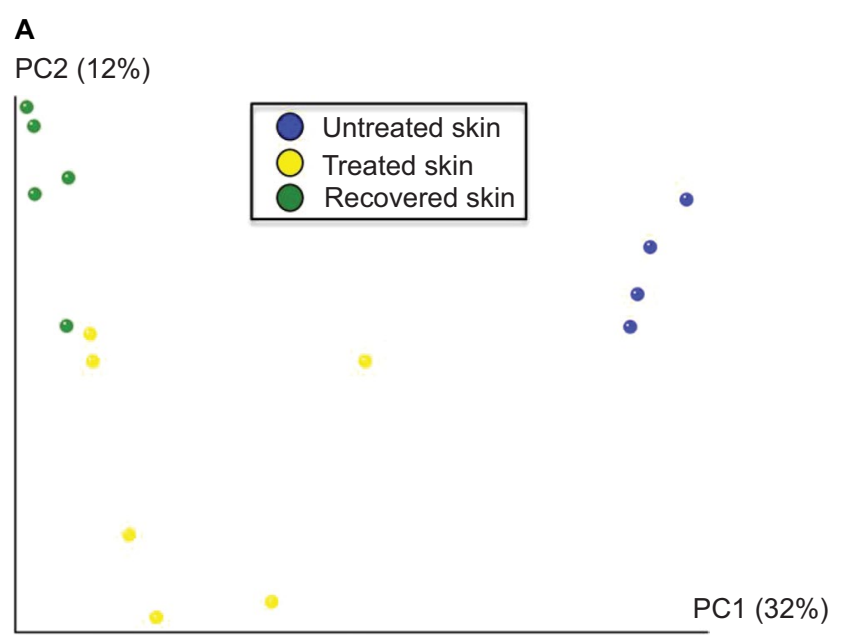

B

PC2 (15\%)

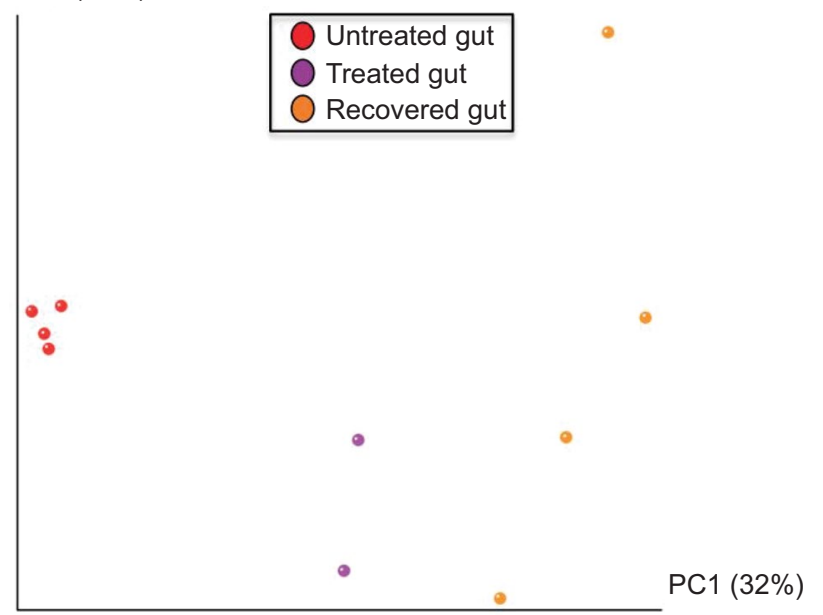

Figure 6 Recovered skin and gut microbiomes do not return to pretreated composition Unweighted UniFrac-based principal components analysis. (A) Skin and (B) gut. Abbreviation: PC, principal components analysis.

is $\sim 0.1 \%-0.01 \%$ of the culturable population) and selected by the antibiotic exposure. In this case, the genus Myroides was selected earliest and to the greatest degree. Rifampicin resistance was expected, but resistance to ceftriaxone and trimethoprim/sulfamethoxazole also appeared, for as yet unclear reasons. There is very little literature on the susceptibilities of Myroides spp., and none on fish-derived strains. Three patients with urinary tract infections from Myroides odoratimimus were successfully treated with a combination of ciprofloxacin and rifampicin. ${ }^{20}$ Myroides spp. are noted to be commonly found in aquatic environments, and can be opportunistic pathogens..$^{21}$ Myroides organisms are reported to often be intrinsically resistant to $\beta$-lactam antibiotics. It is unclear at this time if the Myroides spp. present in fish used in this study are acting as a commensal or are pathogenic.
An interesting result in this study was the loss of Rickettsia from the fish. In the skin microbiome before treatment, the genus Rickettsia represented $6.04 \%$ of all sequences. This was the only genus recovered in the Rickettsiaceae family, whereas 14 OTUs were present in the order Rickettsiales, comprising $32.9 \%$ of all sequences $(25.6 \%$ coming from two OTUs of Captivus). Thus, these organisms are a dominant component in the normal fish skin microbiome, with Rickettsia being the third-most abundant genus, and Rickettsiales counting for almost one-third of all sequences. At the first treatment timepoint of $50.5 \mathrm{~h}$, Rickettsia had dropped to $0.25 \%$ and the Rickettsiales were down to $6.11 \%$. At the last timepoint of $161.5 \mathrm{~h}$, Rickettsia was at only $0.013 \%$ and Rickettsiales was at only $0.093 \%$, and contained only five OTUs, a drastic loss of abundance and diversity. Across the five fish during the week of post-antibiotic recovery, no Rickettsia sequences were found and Rickettsiales only comprised an average of $0.013 \%$ of all sequences. Likewise, the Rickettsia genus was present in the four control fish gut samples at an average of $0.073 \%$, declined in the treatment samples to $0.003 \%$, and was at $0.0004 \%$ in the first recovery timepoint of $20.5 \mathrm{~h}$, and lacking in the latter two recovery points. Rifampicin cleared Rickettsia from the fish skin and gut. Given that Rickettsia is an obligate intracellular organism, this highlights the ability of rifampicin to reach this niche. Whether Rickettsia plays a pathogenic, commensal, or other role with the fish is unclear at this point in the literature.

The API-based approach to examine community biochemical activities is limited to organisms that can live in those culture conditions, so it may not capture all of the biodiversity present. Thus, it determines the biochemical capabilities of community members, but not certainty that those pathways are active in the mucosal microbiome environment. The alignment of API-derived biochemical results with community $16 \mathrm{~S}$-derived identifications is unfeasible, because prediction of biochemical profiles from genuslevel identification is too variable. Although methods are developing for predicting the biochemical pathways in a community from only $16 \mathrm{~S}$ profile data, such as PICRUSt (phylogenetic investigation of communities by reconstruction of unobserved states), ${ }^{22}$ this bioinformatic approach is based on genomic data of human-derived strains, and lacks power with the fish-derived organisms of this study. However, the net loss of four of the community API biochemical activities at 3 days of treatment is consistent with the diversity loss observed by the $16 \mathrm{~S}$ sequencing data.

In mice treated with one of three different antibiotics (clindamycin, ampicillin, or enrofloxacin) for 2 days, 
susceptibility to a following dose of $C$. difficile varied from high to moderate to low, respectively. ${ }^{23}$ This suggests that individual antibiotics affect a key function of the normal microbiome, namely colonization resistance, very differently. The controllable vertebrate model used in this study is now being used to investigate the effects on the host organism resulting from significant antibiotic-induced microbiome community changes, ${ }^{24,25}$ and driving factors during the recovery of the microbiome after antibiotic stress. A 3-day exposure of fish to rifampicin at the same concentration and same method used in this study resulted in reduced resistance to the pathogen Edwardsiella ictaluri, reduced weight gain over a month, and increased susceptibility to osmotic stress, compared to controls. The mechanisms behind these negative effects are being investigated.

\section{Disclosure}

The authors report no conflicts of interest in this work.

\section{References}

1. Ferrer M, Méndez-Garcia C, Rojo D, Barbas C, Moya A. Antibiotic use and microbiome function. Biochem Pharmacol. In press 2016.

2. Dethlefsen L, Relman DA. Incomplete recovery and individualized responses of the human distal gut microbiota to repeated antibiotic perturbation. Proc Natl Acad Sci USA. 2011;108:4554-4561.

3. Dethlefsen L, Huse S, Sogin ML, Relman DA. The correct pervasive effects of an antibiotic on the human gut microbiota, as revealed by deep 16S rRNA sequencing. PLoS Biol. 2008;6:e280.

4. Fouhy F, Guinane CM, Hussey S, et al. High-throughput sequencing reveals the incomplete, short-term recovery of infant gut microbiota following parenteral antibiotic treatment with ampicillin and gentamicin. Antimicrob Agents Chemother. 2012;56:5811-5820.

5. Antonopoulos DA, Huse SM, Morrison HG, Schmidt TM, Sogin ML, Young VB. Reproducible community dynamics of the gastrointestinal microbiota following antibiotic perturbation. Infect Immun. 2009; 77:2367-2375.

6. Leonard AB, Carlson JM, BishoffDE, et al. The skin microbiome of Gambusia affinis is defined and selective. Adv Microbiol. 2014;4:335-343.

7. Drugs.com [webpage on the Internet]. Rifampin Dosage. Available from http://www.drugs.com/dosage/rifampin.html.

8. Caporaso JG, Lauber CL, Walters WA, et al. Ultra-high-throughput microbial community analysis on the Illumina HiSeq and MiSeq platforms. ISME J. 2012;6:1621-1624.

9. Caporaso JG, Kuczynski J, Stombaugh J, et al. QIIME allows analysis of high-throughput community sequencing data. Nat Methods. 2010; $7: 335-336$.
10. Edgar RC. Search and clustering orders of magnitude faster than BLAST. Bioinformatics. 2010;26:2460-2461.

11. Human Microbiome Project Consortium. Structure, function and diversity of the healthy human microbiome. Nature. 2012;486:207-214.

12. Integrative HMP (iHMP) Research Network Consortium. The Integrative Human Microbiome Project: dynamic analysis of microbiome-host omics profiles during periods of human health and disease. Cell Host Microbe. 2014;16:276-289.

13. Wong CH, Hendrix M, Priestley ES, Greenberg WA. Specificity of aminoglycoside antibiotics for the A-site of the decoding region of ribosomal RNA. Chem Biol. 1998;5:397-406.

14. Mehta R, Champney WS. 30 S ribosomal subunit assembly is a target for inhibition by aminoglycosides in Escherichia coli. Antimicrob Agents Chemother. 2002;46:1546-1549.

15. Lee CK, Herbold CW, Polson SW, et al. Groundtruthing next-gen sequencing for microbial ecology-biases and errors in community structure estimates from PCR amplicon pyrosequencing. PLoS One. 2012; 7:e44224.

16. Roeselers G, Mittge EK, Stephens WZ, et al. Evidence for a core gut microbiota in the zebrafish. ISME J. 2011;5:1595-1608.

17. Brooks AW, Kohl KD, Brucker RM, van Opstal EJ, Bordenstein SR. Phylosymbiosis: relationships and functional effects of microbial communities across host evolutionary history. PLoS Biol. 2016;14:e2000225.

18. Navarrete P, Mardones P, Opazo R, Espejo R, Romero J. Oxytetracycline treatment reduces bacterial diversity of intestinal microbiota of Atlantic salmon. J Aquat Anim Health. 2008;20:177-183.

19. Lowrey L, Woodhams DC, Tacchi L, Salinas I. Topographical mapping of the Rainbow Trout (Oncorhynchus mykiss) microbiome reveals a diverse bacterial community with antifungal properties in the skin. Appl Environ Microbiol. 2015;81:6915-6925.

20. Ktari S, Mnif B, Koubaa M, et al. Nosocomial outbreak of Myroides odoratimimus urinary tract infection in a Tunisian hospital. J Hosp Infect. 2012;80:77-81.

21. Mammeri H, Bellais S, Nordmann P. Chromosome-encoded $\beta$-lactamases TUS-1 and MUS-1 from Myroides odoratus and Myroides odoratimimus (formerly Flavobacterium odoratum), new members of the lineage of molecular subclass B1 metalloenzymes. Antimicrob Agents Chemother. 2002;46:3561-3567.

22. Langille MGI, Zaneveld J, Caporaso JG, et al. Predictive functional profiling of microbial communities using 16S rRNA marker gene sequences. Nat Biotechnol. 2013;31:814-821.

23. Buffie CG, Bucci V, Stein RR, et al. Precision microbiome reconstitution restores bile acid mediated resistance to Clostridium difficile. Nature. 2015;517:205-208.

24. Carlson JM, Hyde ER, Petrosino JF, Manage AB, Primm TP. The host effects of Gambusia affinis with an antibiotic-disrupted microbiome. Comp Biochem Physiol C Toxicol Pharmacol. 2015;178:163-168.

25. Carlson JM, Chavez O, Aggarwal S, Primm TP. Examination of host phenotypes in Gambusia affinis following antibiotic treatment. JVis Exp. 2017; 120:e55170.

26. Glöckner FO, Zaichikov E, Belkova N, et al. Comparative $16 \mathrm{~S}$ rRNA analysis of lake bacterioplankton reveals globally distributed phylogenetic clusters including an abundant group of actinobacteria. Appl Environ Microbiol. 2000;66:5053-5065.
Infection and Drug Resistance

Publish your work in this journal

Infection and Drug Resistance is an international, peer-reviewed openaccess journal that focuses on the optimal treatment of infection (bacterial, fungal and viral) and the development and institution of preventive strategies to minimize the development and spread of resistance. The journal is specifically concerned with the epidemiology of antibiotic

\section{Dovepress}

resistance and the mechanisms of resistance development and diffusion in both hospitals and the community. The manuscript management system is completely online and includes a very quick and fair peerreview system, which is all easy to use. Visit http://www.dovepress.com/ testimonials.php to read real quotes from published authors. 